\title{
Do concomitant pain symptoms in patients with major depression affect quality of life even when taking into account baseline depression severity?
}

This article was published in the following Dove Press journal:

Patient Preference and Adherence

24 May 2013

Number of times this article has been viewed

\section{Diego Novick' \\ William Montgomery ${ }^{2}$ \\ Zbigniew Kadziola ${ }^{3}$ \\ Victoria Moneta ${ }^{4}$ \\ Xiaomei Peng 5 \\ Roberto Brugnoli ${ }^{6}$ \\ Josep Maria Haro ${ }^{4}$ \\ 'Eli Lilly and Company, Windlesham, Surrey, UK; ${ }^{2}$ Eli Lilly Australia Pty Ltd, West Ryde, NSW, Australia; ${ }^{3}$ Eli Lilly Austria $\mathrm{GmbH}$, Vienna, Austria; ${ }^{4}$ Parc Sanitari Sant Joan de Déu, Fundació Sant Joan de Déu, CIBERSAM, Universitat de Barcelona, Barcelona, Spain; ${ }^{5}$ Eli Lilly and Company, Indianapolis, IN, USA; 'Università di Roma, "Sapienza," Rome, Italy}

Correspondence: Diego Novick Lilly Research Centre, Erl Wood Manor, Sunninghill Road, Windlesham, Surrey GU20 6PH, UK

Tel +44 I27 6483832

Fax +44 I276483192

Email novick_diego@lilly.com
Background: Patients with major depressive disorder (MDD) may suffer from concomitant pain symptoms. The aim of this study is to determine whether the presence of painful physical symptoms (PPS) influences quality of life when taking into account baseline depression severity.

Methods: Patients with a new or first episode of MDD $(n=909)$ were enrolled in a 3-month prospective observational study in East Asia. The Hamilton Depression Rating Scale, Clinical Global Impression-Severity score, Somatic Symptom Inventory, and EuroQoL questionnaire-5 Dimensions (EQ-5D) and EQ-Visual Analogue Scale (EQ-VAS) were assessed at baseline and 3 months' follow-up. The presence of PPS was defined as a mean score of $\geq 2$ on the Somatic Symptom Inventory pain-related items. Regression analyses determined predictors of quality of life at 3 months, adjusting for age, sex, depressive symptoms, overall severity, and quality of life at baseline.

Results: PPS were present (PPS+) at baseline in 52\% of patients. During the 3-month follow-up, EQ-VAS scores improved from 47.7 (standard deviation [SD] 20.6) to 72.5 (SD 20.4), and EQ-5D improved from 0.48 (SD 0.34) to 0.80 (SD 0.26). At 3 months, mean EQ-VAS was 66.4 (SD 21.2) for baseline PPS+ patients versus 78.5 (SD 17.6) for baseline PPS- patients, and mean EQ-5D was 0.71 (SD 0.29) versus 0.89 (SD 0.18). PPS+ at baseline was a significant predictor of quality of life at 3 months after adjusting for sociodemographic and baseline clinical variables.

Conclusion: The presence of painful physical symptoms is associated with less improvement in quality of life in patients receiving treatment for major depression, even when adjusting for depression severity.

Keywords: pain, depression, quality of life, treatment, Asia, course

\section{Introduction}

Major depressive disorder (MDD) is a common and debilitating condition that rates among the highest causes of disability in the world. ${ }^{1}$ The average 12 -month prevalence of MDD has been estimated to be around 5.5\% in ten high-income countries, and $5.9 \%$ in eight low- to middle-income countries. ${ }^{2}$ The diagnostic criteria for MDD is mainly based in psychological and vegetative symptoms. However, other somatic and pain symptoms are frequent in these patients. ${ }^{3}$ Moreover, somatic or pain symptoms are usually the main reason for a depressed patient's initial visit to the primary care physician. $^{4}$

Painful physical symptoms (PPS), including headaches, stomach pain, back pain, and vague, poorly localized pain, are frequent in patients with depression. ${ }^{4-8}$ 
The presence of pain predicts a longer time to remission, ${ }^{9}$ and the resolution of these physical symptoms is a strong predictor of full remission. ${ }^{10}$ Failure to address the physical symptoms associated with depression may compromise the overall remission rate. ${ }^{7,11,12}$ Also, patients who achieve remission but continue to suffer from residual physical symptoms may have a greater risk of clinical relapse. ${ }^{13}$

Patients with depression rate their quality of life (QoL) lower than the general population or lower than patients with other chronic diseases, such as diabetes, arthritis, or cardiovascular disease. ${ }^{14-17}$ Although QoL improves after starting antidepressant medication, it remains low compared to healthy controls, ${ }^{18}$ and several depression-related variables, including initial severity of depression, have been associated with worse QoL outcomes in depressed patients. ${ }^{19}$ Coexisting pain symptoms are also known to be associated with poorer outcomes, including worse patient-reported QoL, lower productivity, and increased health care utilization. ${ }^{20}$

The association between depression and pain is complex, and the underlying mechanisms are not yet fully understood, but they are known to share a common neurochemical pathway such that both pain and depression are influenced by serotonin and norepinephrine..$^{20,22-24}$ It has been suggested that the differential clinical phenomenology in depression is caused by dysfunction of specific neural pathways modulated by serotonin and norepinephrine. ${ }^{25}$ This seems to be true for both psychological and somatic symptoms of depression. ${ }^{24}$ A key clinical question is whether physicians need to take into account pain symptoms when diagnosing or treating a patient with major depression. If both pain and mood symptoms in depression have a common mechanism, then treating mood symptoms alone would be sufficient and pain would have a negligible impact on outcomes. Conversely, if pain and mood symptoms have different underlying mechanisms, pain symptoms may not impact outcomes through depression severity, hence, this should be taken into account in the treatment of the patient.

The aim of this observational study of Asian patients treated for an acute episode of MDD is to examine whether the presence of PPS influences patient self-reported QoL even when adjusting for the severity of depression.

\section{Methods}

\section{Study design and participants}

This 3-month, prospective, observational study in the psychiatric care setting enrolled 909 patients from 30 study sites across six East Asian countries and regions: China (Mainland), Hong Kong, Korea, Malaysia, Singapore, and Taiwan. Patients were recruited from June 14, 2006 to February 15, 2007, and then they were followed for a period of 3 months. The study was reviewed and approved by the institutional or ethical review board of at least one site in each participating country/region. Written informed consent was obtained from all patients or their legal representative prior to enrolment.

Patients included in the study were inpatients and outpatients at least 18 years of age, who presented with a new or first episode of MDD, as defined by the diagnostic criteria of the Diagnostic and Statistical Manual of Mental Disorders, Fourth Edition Text Revision, ${ }^{26}$ or the International Classification of Diseases, Tenth Revision (ICD-10). ${ }^{27}$ Additional inclusion criteria were: Clinical Global Impressions Severity of Illness (CGI-S) score $\geq 4$ (moderate) at study entry; ${ }^{28}$ at least 2 months free of depression symptoms prior to the onset of the present episode; and consent to participate. Patients were excluded if their current depressive episode had persisted for more than 6 continuous months; if they had a previous or current diagnosis of schizophrenia, schizophreniform disorder, schizoaffective disorder, bipolar disorder, or dementia; if they were experiencing chronic treatment-resistant pain or pain of an inflammatory origin related to an identified medical condition; or were simultaneously participating in another study that included a treatment intervention and/or an investigational drug.

All treatment decisions and provision of care to study participants with MDD were based solely on each health care provider's usual practice and was independent of participation in the study. Adverse events were reported to the corresponding health authorities as per each country's local rules, regulations, and legislation. The study was conducted in accordance with the ethical principles that have their origin in the Declaration of Helsinki and are consistent with the International Conference on Harmonization good clinical practice guidelines.

\section{Measures}

A full description of the data collected at baseline and during the study, including demographic and clinical data, has been reported previously. ${ }^{21,29}$

The severity of depression was assessed at baseline and at 3 months using the CGI-S and the 17-item Hamilton Depression Rating Scale (HAMD-17). ${ }^{30}$ The HAMD-17 total score ranges from $0-52$, with a higher score indicating more severe depression. Response was defined as a decrease of at least $50 \%$ in the HAMD-17 total score from baseline to 
endpoint. Remission was defined as a HAMD-17 total score of $\leq 7$ at the study endpoint.

Patients were assessed at baseline for either the presence or absence of PPS (PPS+ and PPS-, respectively). PPS+ was defined as a mean score of $\geq 2$ for the seven pain-related items of the Somatic Symptom Inventory (SSI), which included abdominal pain, lower back pain, joint pain, neck pain, pain in heart or chest, headaches, and muscular soreness. ${ }^{31}$ Patients rated the degree to which each symptom bothered them over the past week on a scale of 1 (not at all) to 5 (a great deal).

Patient perception of QoL was assessed using the EuroQoL Questionnaire-5 Dimensions (EQ-5D). ${ }^{32}$ This instrument has five items (mobility, self-care, usual activities, pain/discomfort, and anxiety/depression), each of which is scored on a scale from 1 (no problems) to 3 (extreme problems). The responses are converted to a "utility" score that ranges from $0-1$, where 0 represents deceased and 1 represents perfect health. The UK tariff was applied to the EQ-5D data of the Asian patients to calculate the utility score. ${ }^{33}$ The EQ-5D questionnaire also includes a visual analogue scale (EQ-VAS), on which patients are asked to rate their current overall health that day on a scale from 0 (worst imaginable health state) to 100 (best imaginable health state), thus providing an overall "health state" score.

Data involving treatment patterns, including antidepressants and other prescribed medications and treatments for MDD and pain, were collected at baseline and during the study period, as reported previously. ${ }^{21,29}$

\section{Statistical analysis}

Data are summarized descriptively, with means and standard deviations (SD) for numerical variables and percentages for categorical variables.

The distribution of QoL scores (EQ-5D and EQ-VAS) at the study endpoint was identified by the presence or absence of PPS at baseline, and four different levels of baseline depression severity were described using box and whisker plots. The HAMD-17 total scores used for the different depression severity levels at baseline were: $\leq 16,16-22$, $22-28$, and $>28$.

Bivariate analysis examined the association between baseline variables and QoL outcomes (EQ-5D and EQ-VAS) at the study endpoint. The association between severity of depression at baseline (CGI-S, HAMD-17) and QoL at both baseline and endpoint (EQ-5D, EQ-VAS) was described using Pearson correlation coefficients.

Multivariable linear regression modeling was used to identify baseline variables (including the presence of PPS) associated with QoL outcomes at 3 months. Dependent variables were the EQ-5D score or EQ-VAS, and the independent variables were the patient demographics (age, sex, marital status, and employment status) and baseline clinical characteristics (depressive symptoms, overall severity, and QoL). Data are presented as parameter estimates and $95 \%$ confidence intervals, with $P$-values.

All statistical analyses were performed using the SAS statistical package version 9.3 (SAS Institute, Inc, Cary, NC, USA).

\section{Results}

The mean age of the total study sample of 909 patients was 45.2 years (SD 14.2) and 69\% were females. Of these patients, $68.5 \%$ were married or had a de facto spouse, while $16.3 \%$ were single, and the remaining $15.2 \%$ were divorced, widowed, or separated. At baseline, $37.5 \%$ of the patients were unemployed, $35.3 \%$ were working full-time, and the remaining $14.6 \%, 7.2 \%$, and $5.4 \%$, respectively, were retired, part-time workers, and students.

Of the 909 patients in the total sample, 471 (51.8\%) had PPS at baseline (PPS+). For the total sample, the baseline mean HAMD-17 total score was 23.7 (SD 5.8) and the baseline mean CGI-S score was 4.7 (SD 0.8), indicating moderate to severe baseline depression symptoms.

Bivariate analysis showed significant correlations between baseline severity of depression (HAMD-17, CGI-S) and QoL (EQ-5D and EQ-VAS) at baseline and at 3 months (Table 1).

\section{Impact of pain on quality of life}

The mean EQ-VAS score improved from 47.7 (SD 20.6) at baseline $(n=907)$ to $72.5(\operatorname{SD} 20.4)$ at endpoint $(n=715)$,

Table I Bivariate correlations between depression severity (HAMD-17, CGI-S) at baseline and quality of life (EQ-5D, EQVAS) at baseline and at the study endpoint

\begin{tabular}{llllll}
\hline & HAMD-I7 & & & CGI-S & \\
\cline { 2 - 3 } \cline { 5 - 6 } & $\begin{array}{l}\text { Correlation } \\
\text { coefficient }\end{array}$ & P-value & & $\begin{array}{l}\text { Correlation } \\
\text { coefficient }\end{array}$ & P-value \\
\hline EQ-5D & & & & & \\
Baseline & -0.401 & 0.001 & -0.408 & $<0.00 I$ \\
Endpoint & -0.131 & 0.0005 & -0.139 & 0.0002 \\
EQ-VAS & & & & \\
Baseline & -0.283 & $<0.001$ & -0.289 & $<0.001$ \\
Endpoint & -0.076 & 0.0433 & -0.052 & 0.166 \\
\hline
\end{tabular}

Note: Data presented are Pearson correlation coefficients.

Abbreviations: HAMD-17, Hamilton Rating Scale for Depression-17; CGI-S, Clinical Global Impression - Severity Scale; EQ-5D, EuroQoL-5 Dimensions; EQ-VAS, EuroQoL-Visual Analogue Scale. 
and the mean EQ-5D score improved from 0.48 (SD 0.34) at baseline $(n=907)$ to $0.80(0.26)$ at endpoint $(n=716)$.

Figures 1 and 2 show that QoL (EQ-VAS and EQ-5D score) at the study endpoint differed between patients with and without PPS at baseline (PPS+, PPS-), irrespective of the baseline severity of depression based on different HAMD-17 total scores. At all depression severity levels, PPS+ patients had lower QoL scores than PPS- patients.

Bivariate analysis showed that the presence of PPS (PPS+) at baseline was a significant predictor of worse patient-rated QoL at 3 months; PPS+ patients had a mean EQ-VAS score of 66.4 (SD 21.2) versus 78.5 (SD 17.6) for PPS- $(P<0.001)$. Likewise, the EQ-5D scores at 3 months were 0.71 (SD 0.29) versus 0.89 (SD 0.18), for the PPS+ and PPS- groups, respectively $(P<0.001)$.

Table 2 summarizes the results of the linear regression models and shows that the presence of PPS at baseline was a significant predictor of QoL at 3 months after adjusting for sociodemographic variables, baseline QoL, and baseline depression severity; PPS+ patients had an EQ-5D score at 3 months that was 0.12 points lower than that of PPSpatients, and an EQ-VAS score that was 9.5 points lower than PPS- patients.
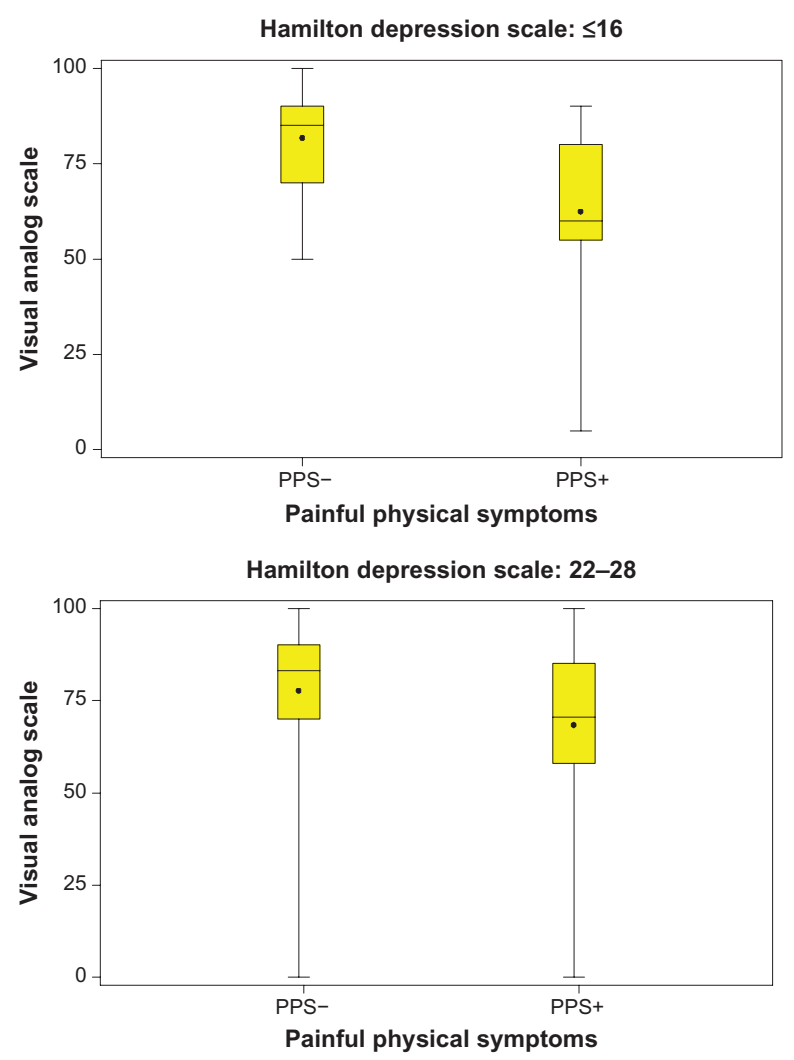

\section{Discussion}

This study of Asian patients treated for an episode of major depression shows that the presence of PPS negatively influences patient QoL even when the severity of depression is taken into account. Specifically, we found that patients with pain symptoms (PPS+) had a lower QoL at baseline and less improvement in their QoL after 3 months of treatment, whether measured by EQ-VAS or EQ-5D score, and when compared with MDD patients without pain symptoms (PPS-). Moreover, after adjusting for baseline covariates (including depression severity), PPS+ was a predictor of QoL at 3 months. Our findings suggest that pain has a greater impact on QoL in patients with MDD than the impact of the severity of depression.

Patients with MDD have a diminished QoL compared with healthy controls, even when in remission. ${ }^{17,18}$ The EQ-5D is a well established and widely used generic instrument for measuring health-related QoL. ${ }^{34}$ The mean EQ-5D utility score at baseline in our sample of patients with an acute episode of MDD (0.48) was similar to that reported in previous studies of depressed patients. ${ }^{14}$ Likewise, the improvement in EQ-5D seen after 3 months of treatment has also been observed in other studies of patients treated with antidepressants. ${ }^{14,17,35}$
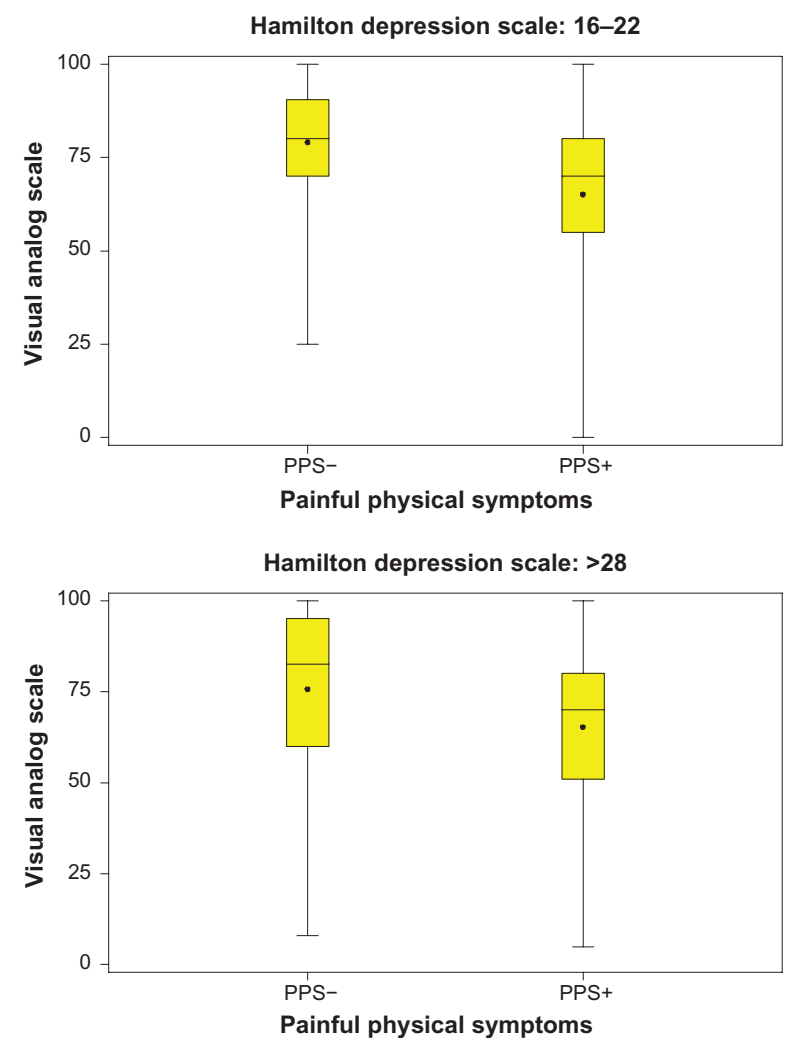

Figure I Influence of baseline PPS on endpoint EQ-VAS is consistent at different severity levels of the HAMD-I7 score at baseline. Abbreviations: PPS, painful physical symptoms; EQ-VAS, EuroQoL-Visual Analogue Scale; HAMD-I7, Hamilton Rating Scale for Depression- 17. 

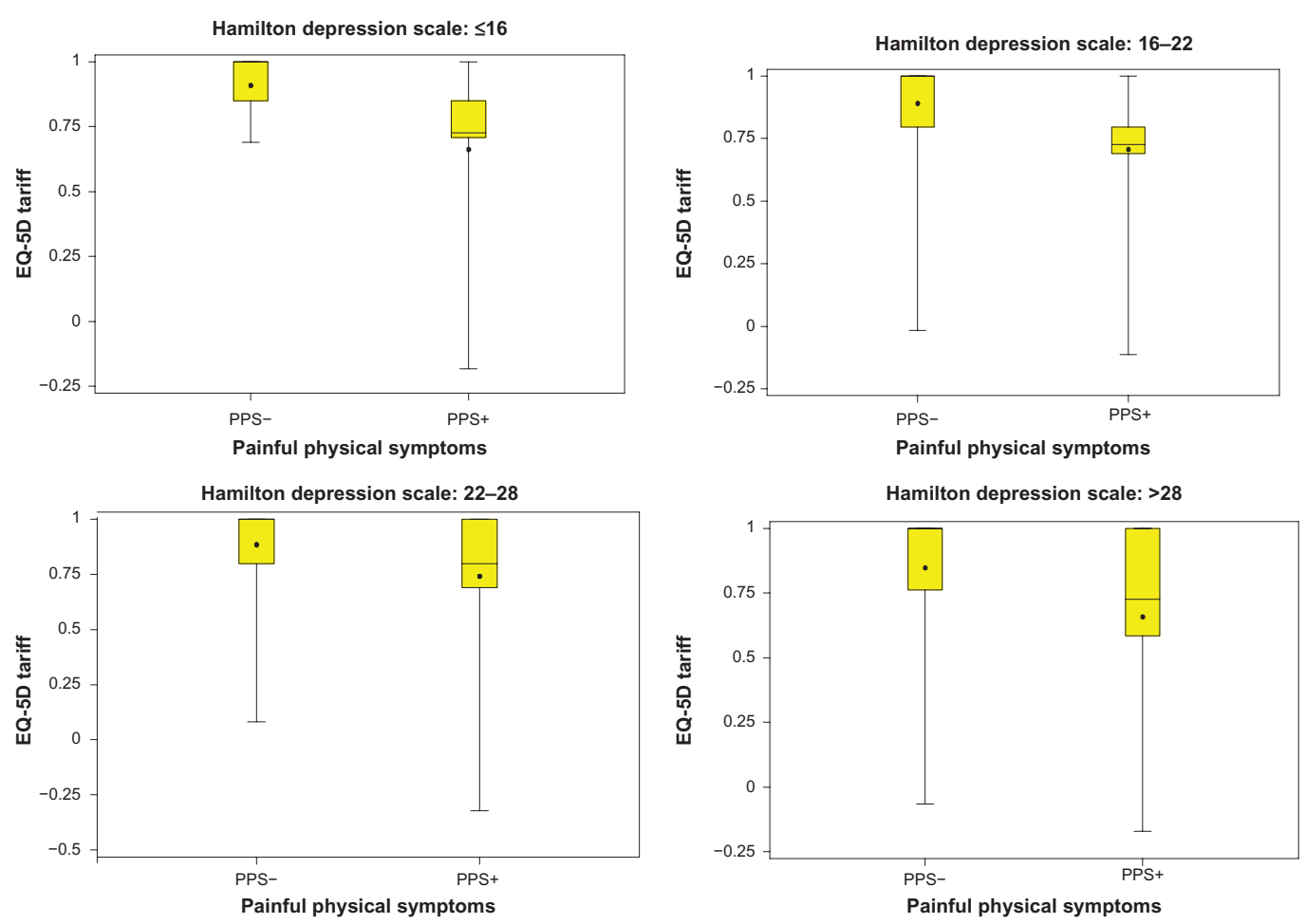

Figure 2 Influence of baseline PPS on endpoint EQ-5D score is consistent at different severity levels of the HAMD-17 score at baseline. Abbreviations: PPS, painful physical symptoms; EQ-5D, EuroQoL-5 Dimension; HAMD- 17, Hamilton Rating Scale for Depression- 17.

The most important finding of our study is that the presence of PPS at baseline has a substantial impact on patient QoL after 3 months of treatment. After adjusting for covariates, the differences in EQ-5D and EQ-VAS scores at 3 months between patients with and without PPS ( 0.12 and 9.5 points, respectively) can be considered to be clinically relevant, as they are above the threshold for an important difference reported by some researchers $(0.05$ or 0.07 for the EQ-5D utility score). ${ }^{35,36}$

Our results are consistent with the findings from previous studies showing that it is the intensity and extent of pain symptoms at baseline that contribute significantly to a less favorable response to depression medication, and to the need for a longer duration of treatment to obtain a satisfying

Table 2 Baseline variables associated with quality of life scores (EQ-5D and EQ-VAS) at 3 months (linear regression analysis)

\begin{tabular}{|c|c|c|c|c|}
\hline \multirow[t]{2}{*}{ Variable } & \multicolumn{2}{|l|}{ EQ-5D } & \multicolumn{2}{|l|}{ EQ-VAS } \\
\hline & Estimate $(95 \% \mathrm{Cl})$ & $P$-value & Estimate $(95 \% \mathrm{Cl})$ & $P$-value \\
\hline Age & $-0.00 \mathrm{I}(-0.003 ; 0.00 \mathrm{I})$ & 0.276 & $-0.208(-0.347 ;-0.069)$ & $0.003 *$ \\
\hline EQ-5D at baseline & $0.197(0.137 ; 0.258)$ & $<0.00 I^{*}$ & & \\
\hline EQ-VAS at baseline & & & 0.145 (0.07I; 0.2।8) & $<0.00 I^{*}$ \\
\hline Baseline HAMD-I 7 total score & $0.001(-0.003 ; 0.004)$ & 0.700 & $0.012(-0.278 ; 0.303)$ & 0.933 \\
\hline Female versus male & $0.026(-0.013 ; 0.065)$ & 0.198 & 1.161 (-2.01I; 4.333) & 0.4723 \\
\hline CGI-S & $-0.002(-0.030 ; 0.026)$ & 0.873 & $0.811(-1.402 ; 3.023)$ & 0.472 \\
\hline Divorced/widowed/separated versus single & $-0.042(-0.118 ; 0.035)$ & 0.283 & $-0.397(-6.616 ; 5.823)$ & 0.900 \\
\hline Married/de facto spouse versus single & $0.011(-0.052 ; 0.074)$ & 0.731 & $5.640(0.5 \mid 3 ; 10.766)$ & $0.031 *$ \\
\hline PPS+ versus PPS- & $-0.121(-0.158 ;-0.084)$ & $<0.00 I^{*}$ & $-9.474(-12.403 ;-6.545)$ & $<0.00 I^{*}$ \\
\hline Full-time versus unemployed & $0.050(0.006 ; 0.094)$ & $0.026^{*}$ & $5.773(2.209 ; 9.338)$ & $0.002^{*}$ \\
\hline Part-time versus unemployed & $0.062(-0.010 ; 0.135)$ & 0.093 & $3.264(-2.698 ; 9.226)$ & 0.283 \\
\hline Retired versus unemployed & $0.121(0.066 ; 0.176)$ & $<0.00 I^{*}$ & II.915 (7.475; 16.354) & $<0.00 I^{*}$ \\
\hline Student versus unemployed & $-0.000(-0.098 ; 0.097)$ & 0.997 & $4.759(-3.165 ; 12.683)$ & 0.239 \\
\hline
\end{tabular}

Notes: Parameter estimates ( $95 \%$ confidence intervals) are presented. *Statistically significant.

Abbreviations: EQ-5D, EuroQoL-5 Dimensions; EQ-VAS, EuroQoL-Visual Analogue Scale; CI, confidence interval; HAMD-I7, Hamilton Rating Scale for Depression-I7; CGI-S, Clinical Global Impression - Severity Scale; PPS, painful physical symptoms. 
result, if at all. ${ }^{7,9,37}$ QoL is an important measure of treatment success in depression. ${ }^{18}$ Previous studies have shown that QoL is decreased in patients with depression and somatic symptoms, ${ }^{38}$ and that greater severity of pain is associated with worse depression, a lower QoL, and a poorer treatment response. ${ }^{7,8}$ The European FINDER study revealed that having fewer somatic symptoms at baseline was associated with better QoL outcomes, whereas more severe pain at baseline (higher pain VAS) was associated with a worse EQ-5D score at 6 months. ${ }^{19}$

The impact of depression severity on QoL in depressed patients with somatic symptoms has not been reported previously, but a few studies have examined the influence of depression severity on QoL. Primary care patients with MDD had significantly lower EQ-5D scores at baseline and during treatment in patients with increasing disease severity, as measured using the CGI-S. ${ }^{35}$ This French study also showed that QoL at 8 weeks' follow-up differed according to patient response to treatment, with responders having a higher EQ-5D score at the study endpoint than nonresponders (0.85 versus 0.58 , respectively). ${ }^{35}$ Among elderly patients with recurrent MDD, an improvement in depression symptoms after 6 weeks of treatment correlated with an improvement in QoL (as measured using the Short Form-36 Health Survey Questionnaire, SF-36), especially in patients who achieved full remission compared with partial responders and nonresponders. ${ }^{38}$ A study of 103 MDD patients in China reported that increasing severity of depression (higher HAMD total score) was significantly associated with a worse QoL at baseline (SF-36). ${ }^{39}$ Also, after 6 weeks of antidepressant treatment, less improvement in QoL was significantly associated with a higher HAMD score at baseline. ${ }^{39}$ However, another study reported few differences in QoL (as measured by SF-36 scores) between patients with different levels of depression severity (based on ICD-10 classifications). ${ }^{40}$

The present results from this large-scale observational study in Asian patients with MDD extend upon the findings that have been reported previously. Lee et $\mathrm{al}^{21}$ showed that PPS+ patients $(51.8 \%$ of the total sample) were more significantly depressed and had a lower QoL than PPSpatients. Moreover, the PPS+ patients have less improvement than the PPS- patients on depression, pain, and QoL measures during 3 months of treatment. ${ }^{29}$ Consistent with our results, a recent study of 414 Korean outpatients with MDD found that $30.4 \%$ had PPS present, and that this PPS+ group had significantly greater depression severity and a lower QoL (EQ-VAS) than the group without PPS. ${ }^{41}$ Thus, there is growing evidence that emphasizes the importance of assessing PPS in patients with depression so that treatments can be targeted to improve outcomes, including patient QoL. Scales commonly used in depression, such as the HAMD-17, have few pain items and, therefore, may underestimate the effect of pain symptoms on both depression and QoL. ${ }^{42}$

Pain and depression have complex pathophysiological mechanisms that are similar, but not yet fully understood. ${ }^{20,22-25}$ The neurochemicals serotonin and norepinephrine are involved in both phenomena, showing that pain and depression are interrelated. ${ }^{43}$ Studies have shown that brain regions involved in the generation of emotion (eg, the medial prefrontal cortex, insular and anterior prefrontal cortex, hypothalamus, and amygdala), send many projections to brainstem structures involved in pain modulation, such as the periaqueductal gray and the rostral-ventromedial medulla. ${ }^{20}$ Despite an overlap in the underlying neurobiological processes of pain and depression, there must be differences because they both contribute to patient QoL.

The clinical implications of this study may be that in the diagnosis and treatment of patients with depression, we should pay close attention to pain symptoms. Epidemiologic studies have found that about $50 \%$ of patients with depression who consult primary care doctors are not appropriately diagnosed. The main reason for the lack of detection of many patients is the reason for consultation. When patients present simultaneously with mood and pain symptoms, they frequently usually complain of the pain symptoms, and the physician may overlook the presence of mood symptoms in these patients. ${ }^{20}$

Besides, when selecting treatment for patients with depression, physicians must determine whether somatic symptoms (especially pain) are present, and they must use treatments that target both the emotional and physical symptoms of depression; this approach should lead to better depression and QoL outcomes for depressed patients who present with PPS. ${ }^{43}$

Some limitations of the present study should be considered when interpreting the results. First, given the observational design of the study, our findings should be interpreted conservatively. Second, somatic symptoms were collected using a specific questionnaire (Somatic Symptom Inventory); however, we do not know how many of the somatic symptoms the patients would have reported of their own accord if not prompted by the questionnaire. Third, patients from primary care were not included in this study; only patients from psychiatric care settings were included. Therefore, our sample is not representative of the total MDD population in these Asian countries and limits the 
generalizability of our findings to primary care patients with major depression. Fourth, comorbidity with anxiety disorders was not assessed, and could be a factor contributing to patient QoL. Fifth, the analyses were based on depression severity and it is unknown whether the same findings would be observed if pain severity had also been taken into account. Additional limitations are that the analysis did not take into account the antidepressant prescribed, which may influence patient QoL ratings at 3 months. Also, we did not assess use of pain relief medication. Finally, because there is no Asian EQ-5D questionnaire, we applied the commonly-used UK version to the EQ-5D data of the Asian patients to calculate the utility scores. ${ }^{33}$ However, there is evidence that different populations value health states differently, including racial/ethnic differences. ${ }^{44}{ }^{46}$ Nevertheless, the EQ-5D has been shown to be useful for assessing QoL in patients with $\mathrm{MDD},{ }^{35}$ and to have acceptable validity and reliability in Asian populations. ${ }^{47}$ In addition, both the EQ-VAS and the EQ-5D utility scores have been shown to be responsive to change in patients with depression. ${ }^{48,49}$

Despite these potential limitations, the results of our study indicate that the presence of PPS is associated with a lower QoL in patients receiving treatment for major depression, and this is not dependent on the severity of depressive symptoms. Our findings imply that clinicians need to take into account the presence of pain symptoms when diagnosing a patient with major depression and when deciding on a treatment strategy for such patients.

\section{Disclosure}

The authors report no conflicts of interest in this work. Diego Novick, William Montgomery, Zbigniew Kadziola, and Xiaomei Peng are employees of Eli Lilly and Company. Jaume Aguado has conducted the statistical analysis under a contract of Fundació Sant Joan de Déu with Eli Lilly and Company. Roberto Brugnoli has acted as a consultant, received grants, and acted as a speaker in activities sponsored by the following companies: BMS, Eli Lilly, and Innovapharma, Sigma-Tau. Josep Maria Haro has acted as consultant or speaker for Astra-Zeneca, Eli Lilly and Company, Lundbeck, and Roche.

\section{References}

1. World Health Organization. The Global Burden of Disease: 2004 Update. Geneva: World Health Organization; 2008.

2. Bromet E, Andrade LH, Hwang I, et al. Cross-national epidemiology of DSM-IV major depressive episode. BMC Med. 2011;9:90.

3. Novick D, Montgomery W, Aguado J, Kadziola Z, Peng X, Brugnoli R, Haro JM. Which somatic symptoms are associated with an unfavorable course in Asian patients with major depressive disorder? J Affect Disord. Epub 2013 Mar 19.
4. Kirmayer LJ, Robbins JM, Dworkind M, Yaffe MJ. Somatization and the recognition of depression and anxiety in primary care. Am J Psychiatry. 1993;150(5):734-741.

5. Min Soo Lee, Sun Young Yum, Jin Pyo Hong, et al. Association between Painful Physical Symptoms and Clinical Outcomes in Korean Patients with Major Depressive Disorder: A Three-Month Observational Study. Psychiatry Investig. 2009;6(4):255-263.

6. Corruble E, Guelfi JD. Pain complaints in depressed inpatients. Psychopathology. 2000;33(6):307-309.

7. Bair MJ, Robinson RL, Eckert GJ, Stang PE, Croghan TW, Kroenke K. Impact of pain on depression treatment response in primary care. Psychosom Med. 2004;66(1):17-22.

8. Muñoz RA, McBride ME, Brnabic AJ, et al. Major depressive disorder in Latin America: the relationship between depression severity, painful somatic symptoms, and quality of life. J Affect Disord. 2005;86(1): 93-98.

9. Karp JF, Scott J, Houck P, Reynolds CF 3rd, Kupfer DJ, Frank E. Pain predicts longer time to remission during treatment of recurrent depression. J Clin Psychiatry. 2005;66(5):591-597.

10. Paykel ES, Ramana R, Cooper Z, Hayhurst H, Kerr J, Barocka A. Residual symptoms after partial remission: an important outcome in depression. Psychol Med. 1995;25(6):1171-1180.

11. Burt VK. Plotting the course to remission: the search for better outcomes in the treatment of depression. J Clin Psychiatry. 2004;65 Suppl 12: $20-25$.

12. Greco T, Eckert G, Kroenke K. The outcome of physical symptoms with treatment of depression. J Gen Intern Med. 2004;19(8):813-818.

13. Paykel ES, Scott J, Teasdale JD, et al. Prevention of relapse in residual depression by cognitive therapy: a controlled trial. Arch Gen Psychiatry. 1999;56(9):829-835.

14. Hays RD, Wells KB, Sherbourne CD, Rogers W, Spritzer K. Functioning and well-being outcomes of patients with depression compared with chronic general medical illnesses. Arch Gen Psychiatry. 1995;52(1):11-19.

15. Wells KB, Stewart A, Hays RD, et al. The functioning and well-being of depressed patients. Results from the Medical Outcomes Study. JAMA. 1989;262(7):914-919.

16. Sobocki P, Ekman M, Agren H, et al. Health-related quality of life measured with EQ-5D in patients treated for depression in primary care. Value Health. 2007;10(2):153-160.

17. ten Doesschate MC, Koeter MW, Bockting CL, Schene AH; for DELTA Study Group. Health related quality of life in recurrent depression: a comparison with a general population sample. J Affect Disord. 2010;120(1-3):126-132.

18. IsHak WW, Greenberg JM, Balayan K, et al. Quality of life: the ultimate outcome measure of interventions in major depressive disorder. Harv Rev Psychiatry. 2011;19(5):229-239.

19. Reed C, Monz BU, Perahia DG, et al. Quality of life outcomes among patients with depression after 6 months of starting treatment: results from FINDER. J Affect Disord. 2009;113(3):296-302.

20. Bair MJ, Robinson RL, Katon W, Kroenke W. Depression and pain comorbidity: a literature review. Arch Intern Med. 2003; 163(20):2433-2445.

21. Lee P, Zhang M, Hong JP, et al. Frequency of painful physical symptoms with major depressive disorder in Asia: relationship with disease severity and quality of life. J Clin Psychiatry. 2009;70(1):83-91.

22. Basbaum AI, Fields HL. Endogenous pain control mechanisms: review and hypothesis. Ann Neurol. 1978;4(5):451-462.

23. Williams LJ, Jacka FN, Pascoe JA, Dodd S, Berk M. Depression and pain: an overview. Acta Neuropsychiatr. 2006;18(2):79-87.

24. Kapfhammer HP. Somatic symptoms in depression. Dialogues Clin Neurosci. 2006;8(2):227-239.

25. Stahl SM. The psychopharmacology of painful physical symptoms in depression. J Clin Psychiatry. 2002;63(5):382-383.

26. American Psychiatric Association. Diagnostic and Statistical Manual of Mental Disorders Text Revision: DSM-IV-TR, 4th ed. Washington, DC: American Psychiatric Association; 2000. 
27. World Health Organization. International Classification of Diseases and Related World Health Problems. Geneva: World Health Organization; 2007.

28. Guy W. ECDEUAssessment Manual for Psychopharmacology, Revised. Bethesda, MD: US Department of Health, Education, and Welfare; 1976.

29. Ang QQ, Wing YK, He Y, et al. Association between painful physical symptoms and clinical outcomes in East Asian patients with major depressive disorder: a 3-month prospective observational study. Int J Clin Pract. 2009;63(7):1041-1049.

30. Hamilton M. A rating scale for depression. J Neurol Neurosurg Psychiatry. 1960;23:56-62.

31. Kroenke K, Spitzer RL, Williams JB, et al. Physical symptoms in primary care. Predictors of psychiatric disorders and functional impairment. Arch Fam Med. 1994;3(9):774-779.

32. EuroQoL - a new facility for the measurement of health-related quality of life. Health Policy. 1990;16(3):199-208.

33. Brooks R, Rabin R, de Charro F, editors. The Measurement and Valuation of Health Status Using EQ-5D: A European Perspective. Dordrecht, The Netherlands: Kluwer Academic Publishers; 2003.

34. Rabin R, de Charro F. EQ-5D: a measure of health status from the EuroQol Group. Ann Med. 2001;33(5):337-343.

35. Sapin C, Fantino B, Nowicki ML, Kind P. Usefulness of EQ-5D in assessing health status in primary care patients with major depressive disorder. Health Qual Life Outcomes. 2004;2:20.

36. Walters SJ, Brazier JE. Comparison of the minimally important difference for two health state utility measures: EQ-5D and SF-6D. Qual Life Res. 2005;14(6):1523-1532.

37. Simon GE, von Korff M, Lin E. Clinical and functional outcomes of depression treatment in patients with and without chronic medical illness. Psychol Med. 2005;35(2):271-279.

38. Doraiswamy PM, Khan ZM, Donahue RM, Richard NE. Quality of life in geriatric depression: a comparison of remitters, partial responders, and nonresponders. Am J Geriatr Psychiatry. 2001;9(4):423-428.
39. Cao Y, Li W, Shen J, Zhang Y. Association between health related quality of life and severity of depression in patients with major depressive disorder. Zhong Nan Da Xue Xue Bao Yi Xue Ban. 2011;36(2):143-148. Chinese.

40. Nuevo R, Leighton C, Dunn G, et al. Impact of severity and type of depression on quality of life in cases identified in the community. Psychol Med. 2010;40(12):2069-2077.

41. Bahk WM, Park S, Jon DI, Yoon BH, Min KJ, Hong JP. Relationship between painful physical symptoms and severity of depressive symptomatology and suicidality. Psychiatry Res. 2011;189(3):357-367.

42. Hung CI, Weng LJ, Su YJ, Liu CY. Depression and somatic symptoms scale: a new scale with both depression and somatic symptoms emphasized. Psychiatry Clin Neurosci. 2006;60(6):700-708.

43. Trivedi MH. The link between depression and physical symptoms. Prim Care Companion J Clin Psychiatry. 2004;6(Suppl 1):12-16.

44. Johnson JA, Luo N, Shaw JW, Kind P, Coons SJ. Valuations of EQ-5D health states: are the United States and United Kingdom different? Med Care. 2005;43(3):221-228.

45. Fu AZ, Kattan MW. Racial and ethnic differences in preference-based health status measure. Curr Med Res Opin. 2006;22(12):2439-2448.

46. Norman R, Cronin P, Viney R, King M, Street D, Ratcliffe J. International comparisons in valuing EQ-5D health states: a review and analysis. Value Health. 2009;12(8):1194-1200.

47. Wang HM, Patrick DL, Edwards TC, Skalicky AM, Zeng HY, Gu WW. Validation of the EQ-5D in a general population sample in urban China. Qual Life Res. 2012;21(1):155-160.

48. Günther OH, Roick C, Angermeyer MC, König HH. The responsiveness of EQ-5D utility scores in patients with depression: A comparison with instruments measuring quality of life, psychopathology and social functioning. J Affect Disord. 2008;105(1-3):81-91.

49. Gerhards SA, Huibers MJ, Theunissen KA, de Graaf LE, Widdershoven GA, Evers SM. The responsiveness of quality of life utilities to change in depression: a comparison of instruments (SF-6D, EQ-5D, and DFD). Value Health. 2011;14(5):732-739.
Patient Preference and Adherence

\section{Publish your work in this journal}

Patient Preference and Adherence is an international, peer-reviewed, open access journal focusing on the growing importance of patient preference and adherence throughout the therapeutic continuum. Patient satisfaction, acceptability, quality of life, compliance, persistence and their role in developing new therapeutic modalities and compounds to

\section{Dovepress}

optimize clinical outcomes for existing disease states are major areas of interest. This journal has been accepted for indexing on PubMed Central. The manuscript management system is completely online and includes a very quick and fair peer-review system. Visit http://www.dovepress.com/ testimonials.php to read real quotes from published authors. 